\title{
Imagens mono e multitemporais Modis para estimativa da área com soja no Estado de Mato Grosso
}

\author{
Tiago Bernardes ${ }^{(1)}$, Marcos Adami(1), Antônio Roberto Formaggio(1), Maurício Alves Moreira(1), \\ Daniela de Azeredo França( ${ }^{(1)}$ e Maikon Roberth de Novaes ${ }^{(2)}$
}

\begin{abstract}
(1)Instituto Nacional de Pesquisas Espaciais, Caixa Postal 515, CEP 12245-970 São José dos Campos, SP. E-mail: bernati@dsr.inpe.br, adami@dsr.inpe.br, formag@dsr.inpe.br, mauricio@dsr.inpe.br, franca@dsr.inpe.br ${ }^{(2)}$ Instituto Brasileiro de Geografia e Estatística, Rua Prudente de Morais, 161, Petrópolis, CEP 59020-400 Natal, RN. E-mail: maikon@ibge.org
\end{abstract}

Resumo - O objetivo deste trabalho foi avaliar uma nova metodologia para mapeamento da cultura da soja no Estado de Mato Grosso, por meio de imagens Modis e de diferentes abordagens de classificação de imagens. Foram utilizadas imagens diárias e imagens de 16 dias. As imagens diárias foram diretamente classificadas pelo algoritmo Isoseg. As duas séries de imagens de 16 dias, referentes ao ciclo total e à metade do ciclo da cultura da soja, foram transformadas pela análise de componentes principais (ACP), antes de serem classificadas. Dados de referência, obtidos por interpretação visual de imagens do sensor TM/Landsat-5, foram utilizados para a avaliação da exatidão das classificações. Os melhores resultados foram obtidos pela classificação das imagens do ciclo total da soja, transformadas pela ACP: índice global de 0,83 e Kappa de 0,63. A melhor classificação de imagens diárias mostrou índice global de 0,80 e Kappa de 0,55. A ACP aplicada às imagens do ciclo total da soja permitiu o mapeamento das áreas de soja com índices de exatidão melhores do que os obtidos pela classificação derivada das imagens de data única.

Termos para indexação: Glycine max, classificação de imagens, componentes principais, estatísticas agrícolas, processamento digital, sensoriamento remoto.

\section{Mono and multitemporal Modis imagery for soybean area estimate in Mato Grosso State, Brazil}

\begin{abstract}
The objective of this work was to evaluate a new methodology to map soybean crop area in Mato Grosso State, Brazil, using Modis imagery and different image classification approaches. Single-day and 16-day images were used. The single-day images were classified using the Isoseg algorithm. Two series of 16-day composite images, covering the full and the half soybean crop cycles, were transformed using principal component analysis (PCA) prior to the classification. A reference data set, achieved by visual interpretation of TM/Landsat-5 images, was used to evaluate the accuracy of the classifications. The best results were reached using the image classification of soybean full cycle, transformed by PCA: overall accuracy of 0.83 , and Kappa of 0.63 . The best single-day classification showed an overall index of 0.80 , and $0.55 \mathrm{Kappa}$. PCA applied to the images of the full cycle allowed for the mapping of soybean crop areas with better accuracy indices than those obtained by the single-day classification.
\end{abstract}

Index terms: Glycine max, image classifications, principal components, agricultural statistics, digital processing, remote sensing.

\section{Introdução}

O mapeamento de áreas plantadas é fundamental para as previsões de safras, necessárias ao monitoramento e às políticas de desenvolvimento agrícola no Brasil. Contudo, é necessário considerar que a alta dinâmica temporal dos cultivos agrícolas exige o uso de dados proporcionalmente variáveis no tempo, bem como o uso de tecnologias eficientes na análise e interpretação de grandes volumes de dados.
Entre as tecnologias disponíveis atualmente, o sensoriamento remoto vem se mostrando apropriado para a geração de informações de suporte ao gerenciamento de agroecossistemas. Assim, as imagens orbitais do sensor Modis ("moderate resolution imaging spectroradiometer") vêm se mostrando eficientes para fins de monitoramento da atividade agrícola, cujos dados, disponíveis desde fevereiro de 2000, são de livre acesso e apresentam características promissoras para mapeamentos de grandes áreas cultivadas, em razão da cobertura global quase

Pesq. agropec. bras., Brasília, v.46, n.11, p.1530-1537, nov. 2011 
diária, qualidade científica e resolução espacial de $250 \mathrm{~m}$ (Justice \& Townshend, 2002).

Ao longo das respectivas estações de cultivo, as culturas agrícolas apresentam padrões dinâmicotemporais peculiares de imagens orbitais e exibem comportamento espectral variável: solo exposto, no período de preparo do solo e plantio; estágios crescentes de biomassa verde, até atingir um máximo vigor vegetativo; e lavoura em senescência. Embora durante algumas fases do seu desenvolvimento as culturas possam assemelhar-se espectralmente a outras classes de cobertura vegetal da superfície terrestre, seu peculiar comportamento espectral, decorrente da dinâmica fenológica, pode ser usado para realçar diferenças em relação às classes fenologicamente mais estáveis ao longo do tempo, como a vegetação nativa. Assim, o uso de séries temporais de imagens de satélite vem sendo crescentemente incluído, com relativo sucesso, nos trabalhos de identificação de culturas agrícolas (Le Hegarat-Mascle et al., 2000; Van Niel \& MacVicar, 2004; Sedano et al., 2005; Galford et al., 2008; Wardlow \& Egbert, 2008; Ozdogan, 2010; Sakamoto et al., 2010).

A soja é uma cultura de verão e, portanto, a presença provável de nuvens pode dificultar a obtenção de imagens orbitais com a frequência temporal desejada (Sano et al., 2007). A resolução temporal quase diária dos dados Modis representa, assim, uma característica de alto interesse, por aumentar significativamente a chance de obtenção de imagens livres de nuvens. No entanto, uma série temporal de imagens contém informação redundante e, consequentemente, elevada correlação entre as imagens (Fritz et al., 2008). O emprego de técnicas de redução da dimensionalidade de dados, como a análise por componentes principais conforme Gonzalez \& Woods (1992), permite a concentração das informações em um número reduzido de imagens com máxima variância. Essa abordagem foi utilizada por Lasaporana (2006), para analisar séries temporais de índices de vegetação.

No Brasil, diversos trabalhos vêm sendo realizados para avaliar o potencial de imagens Modis em estudos com soja (Rizzi et al., 2006, 2009; Rizzi \&Rudorff, 2007; Rudorff et al., 2007; Breunig et al., 2010; Epiphanio et al., 2010). Epiphanio (2007) avaliou o potencial dessas imagens para uso no Estado de Mato Grosso, a partir de metodologias de classificação por superfície de resposta espectro-temporal.
O objetivo deste trabalho foi avaliar uma nova metodologia para mapeamento da cultura da soja no Estado de Mato Grosso, por meio de imagens Modis e diferentes abordagens para a classificação de imagens, com uso de imagens diárias e de 16 dias.

\section{Material e Métodos}

Os dados utilizados neste trabalho corresponderam à área do Estado de Mato Grosso limitada pelas coordenadas $50^{\circ} 00^{\prime} 00^{\prime \prime} \mathrm{W}$ e $62^{\circ} 41^{\prime} 00^{\prime \prime} \mathrm{W} ; 7^{\circ} 00^{\prime} 00^{\prime \prime} \mathrm{S}$ e $18^{\circ} 14^{\prime} 00^{\prime \prime} \mathrm{S}$ (Figura 1). O material de base consistiu das seguintes imagens de satélites e dados temáticos de referência: duas imagens do sensor Modis diárias (produto Mod09), adquiridas entre 21/1/2006 e 24/1/2006; 13 imagens do sensor Modis (produto Mod13) composição com 16 dias, adquiridas entre $15 / 9 / 2005$ e 22/3/2006; e 30 amostras de mapas de cobertura da terra, com as áreas de soja $\left(900 \mathrm{~km}^{2}\right.$ cada, safra 2005/2006), geradas a partir de imagens TM/ Landsat-5 georreferenciadas (Epiphanio, 2007).

Para o processamento de imagens Modis, utilizaramse os aplicativos MRT-Tool (Dwyer \& Schmidt, 2006) e Envi 4.4 (ITT Visual Information Solutions, 2007). Os demais processamentos foram realizados no aplicativo Spring 4.3.3 (Câmara et al., 1996). O sorteio de pontos amostrais e simulação iterativa dos índices

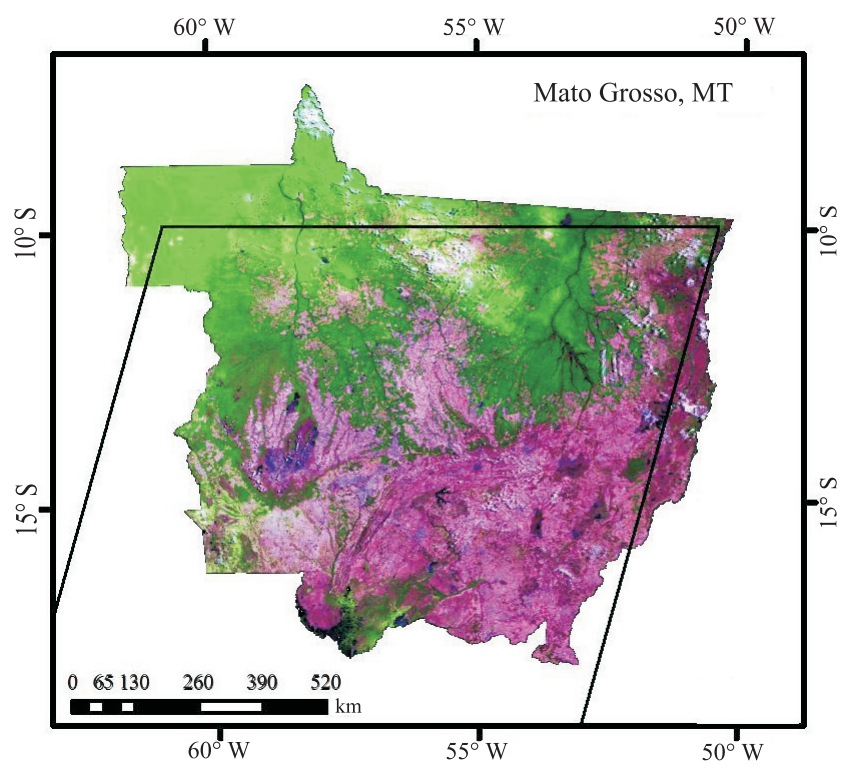

Figura 1. Imagem Modis referente ao Estado de Mato Grosso em composição colorida 1B2G6R. Em detalhe, os limites do tile utilizado, que recobre cerca de $90 \%$ da área total do Estado. 
utilizados na avaliação do desempenho das classificações foram realizados no aplicativo MatLab.

Todas as imagens, obtidas originalmente no formato HDF (hierarchical data format) e projeção sinusoidal, foram processadas inicialmente no aplicativo MRTool ("Modis reprojection tool"), disponibilizado na página do USGS (United States Geological Survey, 2011). Nessa etapa, os dados foram reprojetados para coordenadas latitude/longitude, modelo da terra WGS84 (World Geodetic System 1984) e foram, então, convertidos para o formato GeoTIFF. As etapas de processamento e análise descritas a seguir estão ilustradas esquematicamente na Figura 2.

As imagens Modis monotemporais utilizadas correspondem às bandas $1(0,648 \mu \mathrm{m}), 2(0,858 \mu \mathrm{m})$ e 6 $(1,640 \mu \mathrm{m})$ das cenas diárias, em razão da equivalência espectral com as bandas usadas para a geração dos mapas de referência (TM/Landsat-5). Mesmo com resolução temporal quase diária, não foi possível obter, no período da safra de verão estudada, uma cena única totalmente isenta das nuvens que recobrisse todo o Estado de Mato Grosso. Assim, foi necessário selecionar duas imagens com a menor cobertura de nuvens e melhor geometria de visada a nadir, tendo-se minimizado as distorções geométricas presentes nas bordas da faixa de imageamento (Liesenberg et al., 2007; Breunig et al., 2010). Foi gerada uma máscara por interpretação visual, em cada imagem referente às áreas com ocorrência de nuvens, para a eliminação dessas áreas na classificação. Como as máscaras não se sobrepunham à área eliminada numa imagem, esta foi recoberta pela outra imagem. As imagens foram, então, classificadas separadamente - apenas áreas sem nuvens. Depois de realizadas as classificações, gerou-se um mosaico com os resultados, de modo que as áreas eliminadas numa imagem fossem recobertas por outra imagem, o que resultou em um mapa que cobria a área agrícola do Estado de Mato Grosso. Como a diferença entre as imagens é de apenas três dias, a classificação derivada dessas imagens foi considerada como sendo de uma única data e denominada de Diária.

Para as composições multitemporais (16 dias), foram usadas 13 imagens adquiridas ao longo do período de desenvolvimento fenológico da soja (setembro a março), correspondentes ao "tile H12V10" que cobre $90 \%$ do Estado de Mato Grosso, e uma área de soja correspondente a cerca de $95 \%$ da área total plantada no Estado. A partir desses dados, realizou-se

Processamento de imagens

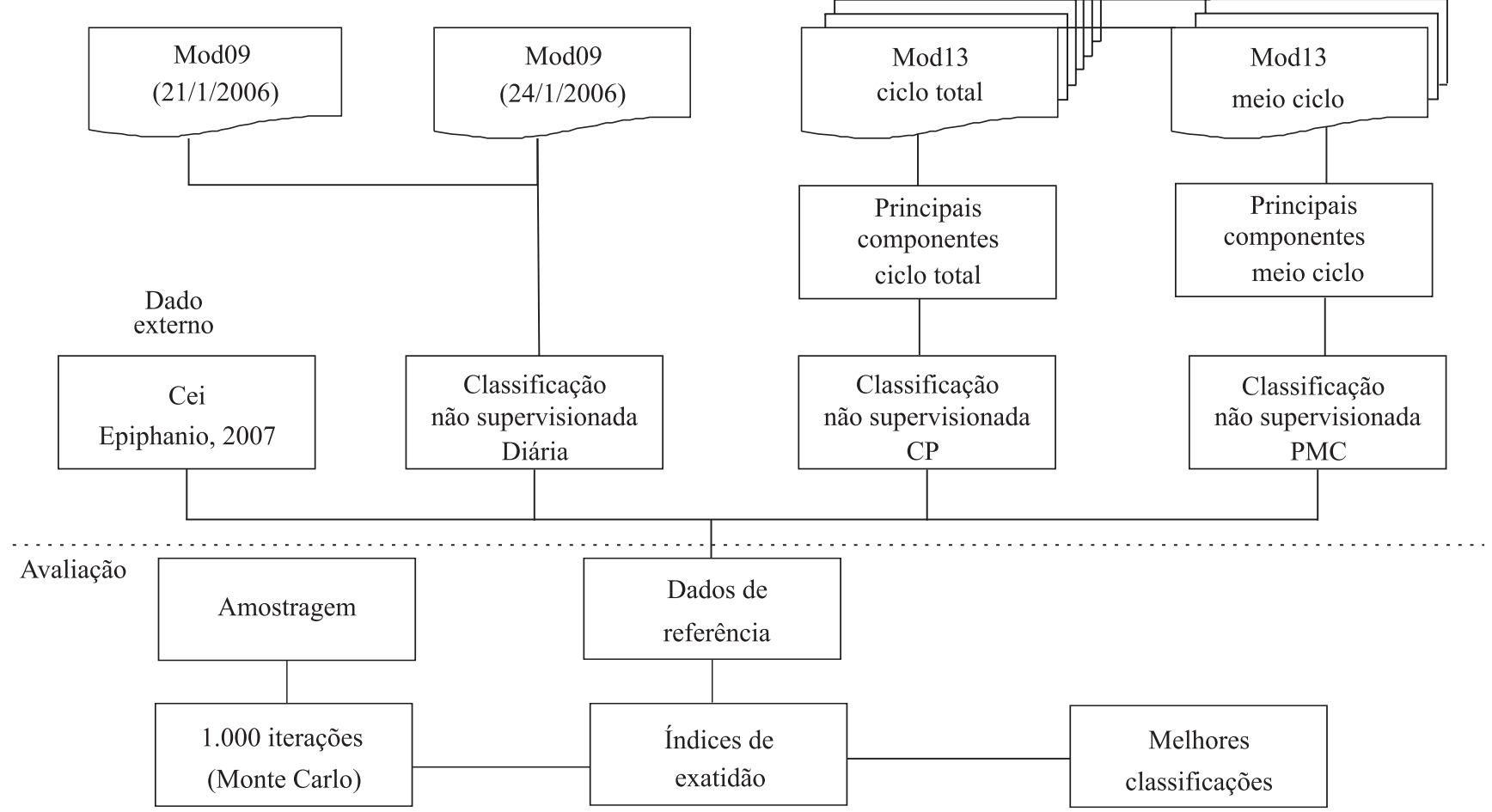

Figura 2. Fluxograma das etapas realizadas nos processamentos e análises dos dados. 
a transformação por componentes principais (CP), para a redução da dimensionalidade dos dados. A transformação foi executada sobre dois conjuntos de imagens: ciclo total, com 52 imagens Modis correspondentes a 13 diferentes datas, com cada data composta de quatro imagens referentes às bandas do azul $(0,470 \mu \mathrm{m})$, vermelho $(0,648 \mu \mathrm{m})$, infravermelho próximo $(0,858 \mu \mathrm{m})$ e infravermelho médio $(1,640 \mu \mathrm{m})$; e meio ciclo, com 28 imagens correspondentes às sete primeiras datas no decorrer do ciclo, com cada data composta pelas mesmas bandas do ciclo total. Após avaliação da matriz de autovalores, foram selecionadas as CP com maior variância para serem utilizadas nas classificações. A partir dessa etapa, apenas as três primeiras componentes (CP1, CP2 e CP3), que continham cerca de $90 \%$ da variabilidade dos dados, foram utilizadas nas classificações. A Figura 3 ilustra a abrangência dos dois conjuntos de imagens utilizados em relação ao ciclo da soja, representado pela curva do índice de realce da vegetação (Evi) para plantio precoce e normal.

As classificações com as $\mathrm{CP}$ derivadas das imagens relativas à primeira metade do ciclo da soja (meio ciclo) foram realizadas para a obtenção de um mapeamento antecipado da área de soja, ou seja, com um caráter preditivo.

Com os dados devidamente processados, foi realizada uma segmentação por crescimento de regiões (Bins et al., 1996) e, em seguida, uma classificação não supervisionada pelo algoritmo Isoseg (Richards,

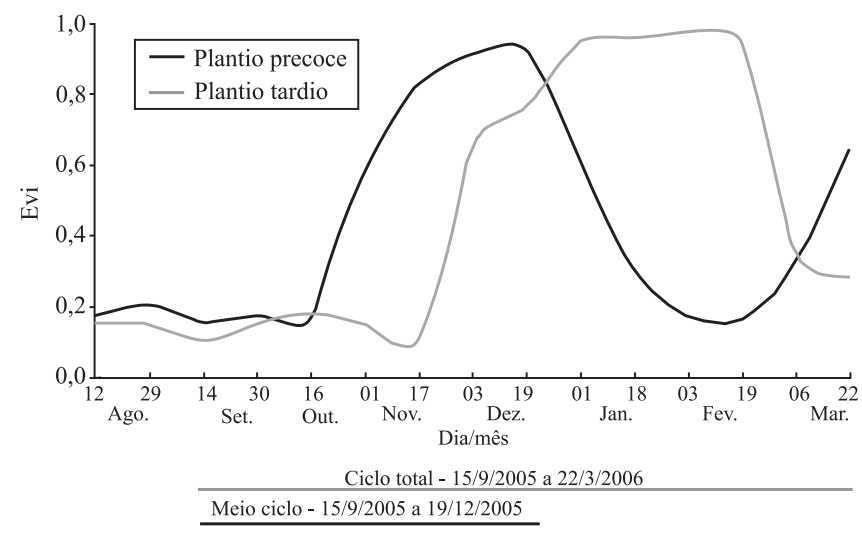

Figura 3. Comportamento temporal do índice de realce da vegetação (Evi), para plantios de soja precoces e tardios em Mato Grosso. Os pontos de máximo Evi representam o período de máximo desenvolvimento vegetativo da soja ao longo do ciclo. Fonte: adaptado de Rizzi et al. (2009).
1995), implementado no sistema Spring (Câmara et al.,1996). Foram utilizadas as duas imagens monotemporais (Diária), bem como as transformações por componentes principais do ciclo total (CPCT) e da primeira metade do ciclo (PMC) das imagens multitemporais. A segmentação foi feita com uso dos mesmos parâmetros para as imagens diárias e componentes principais. Como limiar de similaridade, foram usados oito números digitais (ND) e, como menor região segmentada, usaram-se 128, 64 e 32 pixels, equivalentes a 800,400 e 200 ha, respectivamente. As denominações atribuídas às classificações e os respectivos dados e parâmetros empregados estão apresentados na Tabela 1.

A partir das imagens segmentadas, as áreas foram classificadas como "soja" e "outros", com um limiar de aceitação de $99,9 \%$ para a geração dos mapas.

A avaliação da exatidão dos mapeamentos, gerados pelas imagens Modis, foi realizada pelo sorteio de 100 pontos aleatórios para cada um dos 30 segmentos de referência utilizados. O tamanho amostral foi calculado por meio de uma função binomial, conforme Adami et al. (2005). Esse processo foi repetido 1.000 vezes,

Tabela 1. Denominações atribuídas às classificações, dados utilizados, data/período correspondentes e parâmetros aplicados na segmentação com limiar de área em pixels e limiar de similaridade em números digitais (ND).

\begin{tabular}{|c|c|c|c|c|}
\hline Nome & Imagem & Data/período & $\begin{array}{c}\text { Limiar de área } \\
\text { (pixels) }\end{array}$ & $\begin{array}{c}\text { Similaridade } \\
\text { (ND) }\end{array}$ \\
\hline Diária128 & Diária & 21 e $24 / 1 / 2006$ & 128 & 8 \\
\hline Diária64 & Diária & 21 e $24 / 1 / 2006$ & 64 & 8 \\
\hline Diária32 & Diária & 21 e $24 / 1 / 2006$ & 32 & 8 \\
\hline $1 \mathrm{CP} 128$ & $1 \underline{a} \mathrm{CP}$ & Ciclo completo & 128 & 8 \\
\hline 1CP64 & $1 \underline{\mathrm{a}} \mathrm{CP}$ & Ciclo completo & 64 & 8 \\
\hline $1 \mathrm{CP} 32$ & $1 \underline{a} \mathrm{CP}$ & Ciclo completo & 32 & 8 \\
\hline $2 \mathrm{CP} 128$ & 1 a $2^{\mathrm{a}} \mathrm{CP}$ & Ciclo completo & 128 & 8 \\
\hline 2CР64 & 1 e $2^{a} \mathrm{CP}$ & Ciclo completo & 64 & 8 \\
\hline $2 \mathrm{CP} 32$ & $1^{\mathrm{a}} \mathrm{e} 2^{\mathrm{a}} \mathrm{CP}$ & Ciclo completo & 32 & 8 \\
\hline $3 \mathrm{CP} 128$ & $1^{\mathrm{a}} 2^{\mathrm{a}}$ e $3 \underline{\mathrm{a}} \mathrm{CP}$ & Ciclo completo & 128 & 8 \\
\hline $3 \mathrm{CP} 64$ & $1^{\mathrm{a}} 2^{\underline{a}}$ e $3 \underline{\mathrm{a}} \mathrm{CP}$ & Ciclo completo & 64 & 8 \\
\hline $3 \mathrm{CP} 32$ & $1^{\mathrm{a}} 2^{\mathrm{a}}$ e $3 \underline{\mathrm{a}} \mathrm{CP}$ & Ciclo completo & 32 & 8 \\
\hline 1PMC128 & $1 \underline{a} \mathrm{CP}$ & Meio ciclo & 128 & 8 \\
\hline 1PMC64 & $1 \stackrel{\mathrm{a}}{\mathrm{CP}}$ & Meio ciclo & 64 & 8 \\
\hline 1PMC32 & $1 \stackrel{\mathrm{a}}{\mathrm{CP}}$ & Meio ciclo & 32 & 8 \\
\hline 2PMC128 & $1^{\mathrm{a}}$ e $2^{\mathrm{a}} \mathrm{CP}$ & Meio ciclo & 128 & 8 \\
\hline 2PMC64 & $1^{\mathrm{a}}$ e $2^{\mathrm{a}} \mathrm{CP}$ & Meio ciclo & 64 & 8 \\
\hline 2PMC32 & $1^{\mathrm{a}}$ e $2^{\mathrm{a}} \mathrm{CP}$ & Meio ciclo & 32 & 8 \\
\hline 3PMC128 & $1^{a} 2^{a}$ e $3^{\underline{a}} \mathrm{CP}$ & Meio ciclo & 128 & 8 \\
\hline 3РMC64 & $1^{a} 2^{a}$ e $3^{a} \mathrm{CP}$ & Meio ciclo & 64 & 8 \\
\hline 3РМC32 & $1^{\mathrm{a}} 2^{\mathrm{a}}$ e $3^{\mathrm{a}} \mathrm{CP}$ & Meio ciclo & 32 & 8 \\
\hline
\end{tabular}


por meio de simulação de Monte Carlo, a fim de se obterem 1.000 índices de exatidão para cada método de classificação (global e Kappa). Foram avaliadas as estatísticas descritivas dessas 1.000 simulações num diagrama boxplot para a comparação dos resultados. A estatística Kappa (Congalton \& Green, 1999) resulta numa medida da concordância entre a classificação e o dado de referência, excluída a concordância por chance. Os valores variam de zero, para nenhuma concordância, a um, para concordância total. Landis \& Koch (1977) propuseram as seguintes classes de concordância: $\mathrm{K}>0,8$, forte concordância; $0,4<\mathrm{K}<0,8$, concordância moderada; $\mathrm{K}<0,4$, concordância fraca.

Matrizes de erro foram criadas para cada uma das classificações, para comparar a exatidão entre os mapas classificados e o dado de referência naqueles pontos escolhidos. A análise inclui o emprego dos seguintes termos (Congalton \& Green, 1999): índice de exatidão global (IG), razão entre o número total de pontos classificados corretamente (soma da diagonal principal da matriz) e o número total de pontos amostrados; exatidão do produtor, razão entre o número de pontos corretamente classificados numa determinada classe no mapa estimado e o número de pontos da mesma classe no mapa de referência (complemento: erro de omissão); exatidão do usuário, razão entre o número de pontos corretamente classificados numa determinada classe no mapa estimado e todos os pontos da classe no mesmo mapa (complemento: erro de comissão).

\section{Resultados e Discussão}

As três primeiras componentes derivadas das imagens adquiridas dentro do ciclo total da soja apresentaram $87 \%$ da variabilidade, enquanto as mesmas três componentes derivadas da metade do ciclo apresentaram 90\% da variabilidade. Assim, optou-se por utilizar, nas classificações, apenas as três primeiras componentes, por conterem a maior parte da variabilidade espectral nos períodos relacionados.

A CP1, com $83 \%$ da variância no ciclo total e $82 \%$ da variância no meio ciclo, reflete uma contribuição predominante e homogênea da banda do infravermelho próximo ao longo do tempo, com os maiores autovetores em todas as datas. A CP2, com 3\% da variância no ciclo total e $5 \%$ da variância no meio ciclo, reflete o contraste espectral entre as bandas do infravermelho próximo e Swir ("short wavelength infrared"), o qual aumenta ao longo do tempo, em concordância com o desenvolvimento fenológico da soja, ou seja, quanto maior o desenvolvimento da soja, maior o contraste entre a duas bandas, em virtude do espalhamento da radiação infravermelha pela estrutura foliar e da absorção da radiação no Swir pela água contida na vegetação. A CP3, com 1,6\% da variância no ciclo total e $2,8 \%$ da variância no meio ciclo, foi utilizada apenas para avaliação da sua contribuição no resultado final das classificações, uma vez que apenas a magnitude dos autovalores pode não explicitar possíveis informações úteis contidas nessa componente. De fato, conforme relatado adiante, a incorporação dessa componente afetou significativamente a qualidade das classificações.

No diagrama "boxplot" estão os valores das estimativas do índice Kappa para cada classificação (Figura 4). A classificação 3CP64, com as três primeiras componentes relativas ao ciclo total da soja e segmentação com limiar de área de 64 pixels, gerou os melhores resultados, cuja mediana dos valores dos índices global e Kappa estimados ficou em torno de 0,83 e 0,63 , respectivamente. Dessa classificação, foram omitidos $19 \%$ das áreas de soja existentes no mapa de referência (erro de omissão). Entretanto, 27\% da área total mapeada corresponde a áreas inexistentes no mapa de referência (erro de comissão), o que indica uma tendência de superestimativa das áreas de soja.

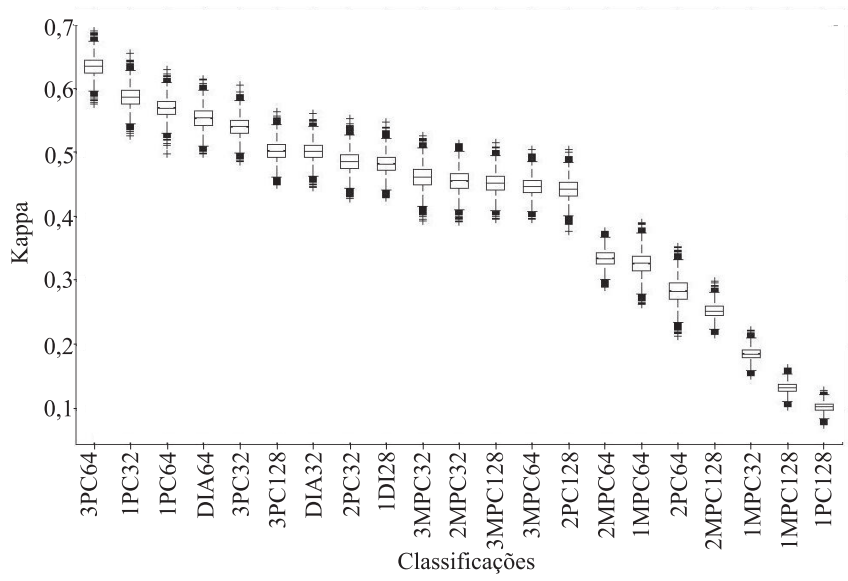

Figura 4. Variação do índice Kappa em 1.000 simulações, para cada classificação. O diagrama "boxplot" ilustra a mediana (linha central), outliers mínimos e máximos (linhas inferiores e superiores) e os quartis: inferior, $25 \%$ dos valores de Kappa; intermediário, retângulo, com 50\% dos valores de Kappa; e superior, 25\% dos valores de Kappa. 
A matriz de confusão obtida para essa classificação está apresentada na Tabela 2.

Em estimativas de áreas de soja, em propriedades do Rio Grande do Sul, com uso de imagens Modis e da metodologia de classificação por superfície de resposta espectro-temporal (STRS), Rudorff et al. (2007) encontraram valores de exatidão global de 0,75 e índice Kappa igual a 0,42. Epiphanio et al. (2010) utilizaram a metodologia STRS sobre imagens Modis e os mesmos segmentos de referência considerados no presente trabalho e obtiveram índices de exatidão global entre 0,80 e 0,86 . Os autores relataram tendência de superestimativa das áreas de soja, em razão do efeito de agregação dos pixels descrito por Soares et al. (2008). Rizzi et al. (2009) inseriram fatores de ganho e realce na formulação do índice Cei, usado por Epiphanio (2007), e obtiveram um valor de exatidão global de 0,91 e erros de omissão e inclusão de $6,1 \%$ e $2,9 \%$, respectivamente.

Deve-se considerar que, a despeito do relativo custo computacional para a transformação por componentes principais e segmentação das imagens, a operacionalização da metodologia para todo o Estado de Mato Grosso é viável, uma vez que o "tile" utilizado recobre a quase totalidade da área do Estado (Figura 1). Ressalta-se ainda que, em virtude do uso das imagens dentro do ciclo completo da cultura, esse mapeamento resulta na estimativa da área de soja somente ao final do ciclo. O mapa com as áreas de soja resultante dessa classificação e os limites das áreas dos mapas de referência para a geração das matrizes de confusão estão apresentados na Figura 5.

As classificações que utilizaram somente a primeira CPCT (ciclo total da soja), com segmentação de $32 \mathrm{e}$ 64 pixels, apresentaram, respectivamente, a segunda e a terceira melhores classificações.

Tabela 2. Matriz de confusão para a mediana das 1.000 simulações, referente à melhor classificação (3CP64).

\begin{tabular}{lccccc}
\hline & Soja & Outros & Total & Comissão Exatidão do usuário \\
\hline Soja & 816 & 309 & 1.125 & 0,27 & 0,73 \\
Outros & 194 & 1.681 & 1.875 & 0,10 & 0,90 \\
Total & 1.010 & 1.990 & 3.000 & \\
Omissão & 0,19 & 0,16 & & \\
Exatidão do produtor & 0,81 & 0,84 \\
\hline Indice global & \multicolumn{5}{c}{0,83} \\
\hline İndice Kappa & \multicolumn{5}{c}{0,63} \\
\hline
\end{tabular}

As classificações com as componentes principais de metade do ciclo (PMC) apresentaram índices Kappa inferiores a 0,50 em todas as simulações. A classificação 2PMC32, derivada das duas primeiras componentes relativas à metade do ciclo da soja e segmentação com limiar de área igual a 32 pixels, apresentou o melhor resultado entre as PMC, com índices global e Kappa iguais a 0,75 e 0,46 , respectivamente. A maior presença de nuvens na primeira metade do ciclo, observada no conjunto original de imagens, pode ter afetado o desempenho dessas classificações. Assim, a possibilidade de obtenção de estimativas antecipadas da área de soja por meio da metodologia proposta mostrou-se não ser a melhor das opções. Para esta finalidade, a classificação DIA64 - imagem de uma única data e limiar de área de 64 pixels - mostrou-se mais adequada, uma vez que apresentou índices de exatidão global e Kappa de 0,80 e 0,55 , respectivamente, com duas imagens tomadas em meados de janeiro, quando a cultura apresenta ainda alta biomassa, ou seja, antes do final do ciclo (Figura 3). Ressalta-se que, para essa classificação, o efeito da presença de nuvens

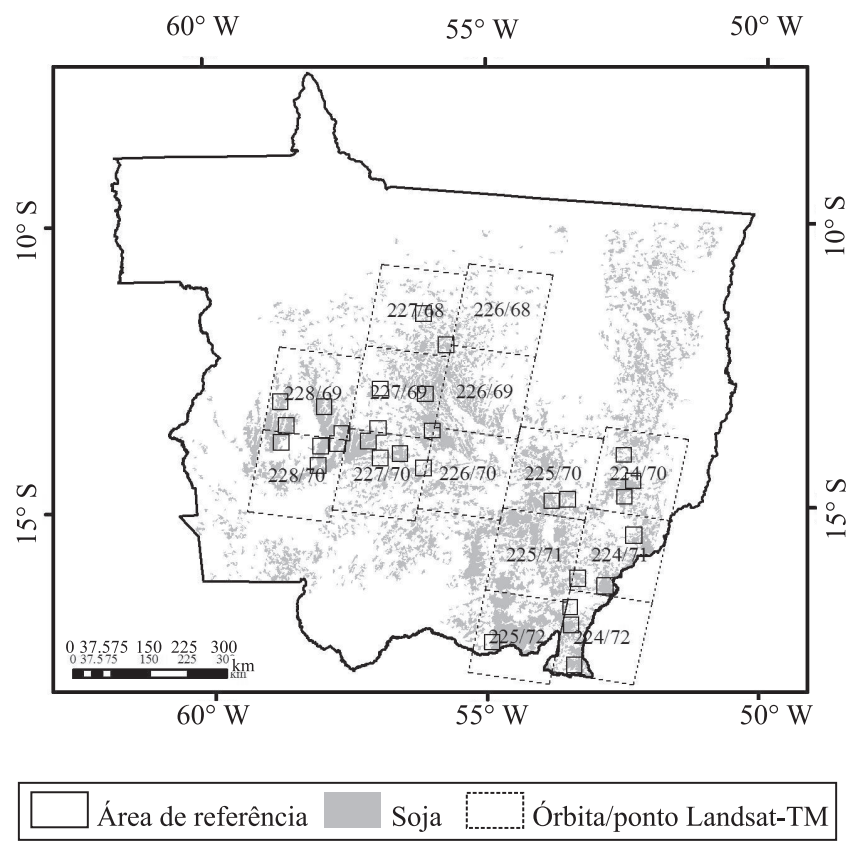

Figura 5. Mapa das áreas de soja do Estado de Mato Grosso, resultante da classificação 3CP64. Em detalhe, posição das áreas dos mapas de referência e órbita/ponto das imagens TM/Landsat-5 usadas para mapeamento de referência (adaptado de Epiphanio, 2007).

Pesq. agropec. bras., Brasília, v.46, n.11, p.1530-1537, nov. 2011 
foi removido com o uso de máscaras e a união das duas imagens, para preencher os espaços originalmente ocupados por nuvens, o que resultou em um ganho na qualidade radiométrica para fins da classificação proposta.

$\mathrm{Na}$ Tabela 3, estão apresentados os índices de exatidão (mediana das mil simulações) em cada classificação, e os valores marginais de acerto da classe soja. De acordo com os níveis de concordância propostos por Landis \& Koch (1977), as 14 melhores classificações enquadram-se na classe de concordância moderada, e sete delas apresentaram concordância fraca. $\mathrm{O}$ presente trabalho refere-se apenas a uma safra de soja no Estado de Mato Grosso. Em anos diferentes, grandes variações nas condições meteorológicas podem resultar em variações na data de plantio, assim como no vigor vegetativo da soja; tais condições podem alterar a resposta espectral da soja e, consequentemente, influenciar os resultados dos classificadores (Wardlow \& Egbert, 2008). Assim, recomenda-se a aplicação da metodologia em múltiplas safras, para a determinação do seu desempenho e sensibilidade dos resultados da classificação à variabilidade interanual da soja no Estado de Mato Grosso.

Tabela 3. Resumo das matrizes de confusão das diferentes classificações e valores marginais de acerto para a classe soja.

\begin{tabular}{lcccccc}
\hline Classificação Índice & Kappa & \multicolumn{3}{c}{ Erro de } & Exatidão do & Erro de \\
global & & omissão & Exatidão do \\
produtor & comissão & usuário \\
\hline 3CP64 & 0,83 & 0,63 & 0,19 & 0,81 & 0,27 & 0,73 \\
1CP32 & 0,81 & 0,59 & 0,25 & 0,75 & 0,28 & 0,72 \\
1CP64 & 0,79 & 0,57 & 0,12 & 0,88 & 0,36 & 0,64 \\
Diária64 & 0,8 & 0,55 & 0,34 & 0,66 & 0,25 & 0,75 \\
3CP32 & 0,78 & 0,54 & 0,17 & 0,83 & 0,36 & 0,64 \\
Diária32 & 0,74 & 0,5 & 0,08 & 0,92 & 0,42 & 0,58 \\
3CP128 & 0,75 & 0,5 & 0,16 & 0,84 & 0,39 & 0,61 \\
2CP32 & 0,75 & 0,49 & 0,18 & 0,82 & 0,41 & 0,59 \\
Diária128 & 0,74 & 0,48 & 0,12 & 0,88 & 0,42 & 0,58 \\
2PMC32 & 0,75 & 0,46 & 0,32 & 0,68 & 0,38 & 0,62 \\
3PMC32 & 0,76 & 0,46 & 0,35 & 0,65 & 0,36 & 0,64 \\
3PMC128 & 0,73 & 0,45 & 0,22 & 0,78 & 0,41 & 0,59 \\
3PMC64 & 0,71 & 0,45 & 0,11 & 0,89 & 0,45 & 0,55 \\
2CP128 & 0,72 & 0,44 & 0,14 & 0,86 & 0,45 & 0,55 \\
2PMC64 & 0,63 & 0,34 & 0,03 & 0,97 & 0,52 & 0,48 \\
1PMC64 & 0,69 & 0,33 & 0,42 & 0,58 & 0,42 & 0,58 \\
2CP64 & 0,68 & 0,28 & 0,48 & 0,52 & 0,46 & 0,54 \\
2PMC128 & 0,57 & 0,25 & 0,02 & 0,98 & 0,55 & 0,45 \\
1PMC32 & 0,52 & 0,19 & 0,02 & 0,98 & 0,58 & 0,42 \\
1PMC128 & 0,47 & 0,13 & 0,01 & 0,99 & 0,6 & 0,4 \\
1CP128 & 0,44 & 0,1 & 0,005 & 0,995 & 0,61 & 0,39 \\
\hline
\end{tabular}

\section{Conclusões}

1. A classificação com uso das três primeiras componentes derivadas das imagens Modis, com recobrimento de toda a janela temporal do ciclo da soja, permite mapear as áreas de soja no Estado de Mato Grosso com melhor exatidão do que a metodologia com a imagem de uma única data.

2. Nas estimativas antecipadas de área de soja em Mato Grosso, o uso de imagens monotemporais, obtidas antes do final do ciclo, apresenta melhores resultados em comparação às imagens referentes à primeira metade do ciclo.

\section{Agradecimentos}

Ao Conselho Nacional de Desenvolvimento Tecnológico e Científico, pelas bolsas concedidas.

\section{Referências}

ADAMI, M.; MOREIRA, M.A.; RUDORFF, B.F.T.; FREITAS, C. da C.; FARIA, R.T. de. Expansão direta na estimativa de culturas agrícolas por meio de segmentos regulares. Revista Brasileira de Cartografia, v.1, p.22-27, 2005.

BINS, L.S.; FONSECA, L.M.G.; ERTHAL, G.J.; MITSUO II, F. Satellite imagery segmentation: a region growing approach. In: SIMPÓSIO BRASILEIRO DE SENSORIAMENTO REMOTO, 8., 1996, Salvador. Anais. São José dos Campos: Inpe, 1996. p.677-680.

BREUNIG, F.M.; GALVÃO, L.S.; FORMAGGIO, A.R.; EPIPHANIO, J.C.N. Directional effects on NDVI and LAI retrievals from MODIS: a case study in Brazil with soybean. International Journal of Applied Earth Observation and Geoinformation, v.13, p.34-42, 2011.

CÂMARA, G.; SOUZA, R.C.M.; FREITAS, U.M.; GARRIDO, J.C.P. SPRING: integrating remote sensing and GIS with object-oriented data modelling. Computers and Graphics, v.15, p.13-22, 1996.

CONGALTON, R.G.; GREEN, K. Assessing the accuracy of remotely sensed data: principles and practices. New York: Lewis, 1999. $137 \mathrm{p}$.

DWYER, J.; SCHMIDT, G. The MODIS reprojection tool. In: QU, J.J.; GAO, W.; KAFATOS, M.; MURPHY, R.E.; SALOMONSON, V.V. (Ed.). Earth science satellite remote sensing. Berlin: Springer, 2006. p.162-177.

EPIPHANIO, R.D.V. Avaliação da potencialidade das imagens MODIS para estimação da área de soja no Estado do Mato Grosso. 2007. 103p. Dissertação (Mestrado) - Instituto Nacional de Pesquisas Espaciais, São José dos Campos.

EPIPHANIO, R.D.V.; FORMAGGIO, A.R.; RUDORFF, B.T.F.; MAEDA, E.E.; LUIZ, A.J.B. Estimating soybean crop areas using 
spectral-temporal surfaces derived from MODIS images in Mato Grosso, Brazil. Pesquisa Agropecuária Brasileira, v.45, p.72-80, 2010.

FRITZ, S.; MASSART, M.; SAVIN, I.; GALLEGO, J.; REMBOLD, F. The use of MODIS data to derive acreage estimations for larger fields: a case study in the south-western Rostov region of Russia. International Journal of Applied Earth Observation and Geoinformation, v.10, p.453-466, 2008.

GALFORD, G.L.; MUSTARD, J.F.; MELILLO, J.; GENDRIN, A.; CERRI, C.C.; CERRI, C.E.P. Wavelet analysis of MODIS time series to detect expansion and intensification of row-crop agriculture in Brazil. Remote Sensing of Environment, v.112, p.576-587, 2008.

GONZALEZ, R.C.; WOODS, R.E. Digital image processing. Reading: Addison-Wesley, 1992. 716p.

ITT VISUAL INFORMATION SOLUTIONS. ENVI: environment for visualizing images. Version 4.4. Boulder: ITT, 2007.

JUSTICE, C.; TOWNSHEND, J. Special issue on the moderate resolution imaging spectroradiometer (MODIS): a new generation of land surface monitoring. Remote Sensing of Environment, v.83, p.1-2, 2002.

LANDIS, J.R.; KOCH, G.G. The measurement of observer agreement for categorical data. Biometrics, v.33, p.159-174, 1977.

LASAPORANA, R. On the use of principal component analysis (PCA) for evaluating interannual vegetation anomalies from SPOT/ VEGETATION NDVI temporal series. Ecological Modelling, v.194, p.429-434, 2006.

LE HEGARAT-MASCLE, S.; QUESNEY, A.; VIDAL-MADJAR, D.; TACONET, O.; NORMAND, M.; LOUMAGNE, C. Land cover discrimination from multitemporal ERS images and multispectral Landsat images: a study case in an agricultural area in France. International Journal of Remote Sensing, v.21, p.435-456, 2000.

LIESENBERG, V.; GALVÃO, L.S.; PONZONI, F.J. Variations in reflectance with seasonality and viewing geometry: implications for classification of Brazilian savanna physiognomies with MISR/Terra data. Remote Sensing of Environment, v.107, p.276-286, 2007.

OZDOGAN, M. The spatial distribution of crop types from MODIS data: temporal unmixing using independent component analysis. Remote Sensing of Environment, v.114, p.1190-1204, 2010.

RICHARDS, J.A. Remote sensing digital image analysis: an introduction. Berlin: Springer-Verlag, 1995. 340p.

RIZZI, R.; RISSO, J.; EPIPHANIO, R.D.V.; RUFORFF, B.F.T.; FORMAGGIO, A.R.; SHIMABUKURO, Y.E.; FERNARDES, S.L.
Estimativa da área de soja no Mato Grosso por meio de imagens MODIS. In: SIMPÓSIO BRASILEIRO DE SENSORIAMENTO REMOTO, 14., 2009, Natal. Anais. São José dos Campos: Inpe, 2009. p.387-394.

RIZZI, R.; RUDORFF, B.F.T. Imagens do sensor MODIS associadas a um modelo agronômico para estimar a produtividade de soja. Pesquisa Agropecuária Brasileira, v.42, n.1, p.73-80, 2007.

RIZZI, R.; RUDORFF, B.F.T.; SHIMABUKURO, Y.E.; DORAISWAMY, P.C. Assessment of MODIS LAI retrievals over soybean crop in Southern Brazil. International Journal of Remote Sensing, v.27, p.4091-4100, 2006.

RUDORFF, C. de M.; RIZZI, R.; RUDORFF, B.F.T.; SUGAWARA, L.M.; VIEIRA, C.A.O. Superfícies de resposta espectro-temporal de imagens do sensor MODIS para classificação de área de soja no Estado do Rio Grande do Sul. Ciência Rural, v.37, p.118-125, 2007.

SAKAMOTO, T.; WARDLOW, B.D.; GITELSON, A.A.; VERMA, S.B.; SUYKER, A.E.; ARKEBAUER, T.J. A two-step filtering approach for detecting maize and soybean phenology with time-series MODIS data. Remote Sensing of Environment, v.114, p.2146-2159, 2010.

SANO, E.E.; FERREIRA, L.G.; ASNER, G.P.; STEINKE, E.T. Spatial and temporal probabilities of obtaining cloud-free Landsat images over the Brazilian tropical savanna. International Journal of Remote Sensing, v.28, p.2739-2752, 2007.

SEDANO, F.; GONG, P.; FERRÃO, M. Land cover assessment with MODIS imagery in southern African Miombo ecosystems. Remote Sensing of Environment, v.98, p.429-441, 2005.

SOARES, D. de M.; GALVÃO, L.S.; FORMAGGIO, A.R. Crop area estimate from original and simulated spatial resolution data and landscape metrics. Scientia Agricola, v.65, p.459-467, 2008.

UNITED STATES GEOLOGICAL SURVEY. MODIS reprojection tool. Available at: $<$ https://pdaac.usgs.gov/lpdaac/ tools/modis_reprojection_tool>. Accessed on: 16 Nov. 2011.

VAN NIEL, T.G.; MCVICAR, T.R. Determining temporal windows for crop discrimination with remote sensing: a case study in South-eastern Australia. Computers and Electronics in Agriculture, v.45, p.91-108, 2004.

WARDLOW, B.D.; EGBERT, S.L. Large-area crop mapping using time-series MODIS $250 \mathrm{~m}$ NDVI data: an assessment for the U.S. Central Great Plains. Remote Sensing of Environment, v.112, p.1096-1116, 2008.

$\overline{\text { Received on October } 1^{\text {th }}, 2010 \text { and accepted on September 30, } 2011}$ 